\title{
Statistical Approach to Boar Semen Head Classification Based on Intracellular Intensity Distribution
}

\author{
Lidia Sánchez ${ }^{1}$, Nicolai Petkov ${ }^{2}$, and Enrique Alegre ${ }^{1}$ \\ ${ }^{1}$ Department of Electrical and Electronics Engineering, \\ University of León, \\ Campus de Vegazana s/n, 24071 León, Spain \\ ${ }^{2}$ Institute of Mathematics and Computing Science, \\ University of Groningen, \\ P.O. Box 800, 9700 AV Groningen, The Netherlands \\ lidia@unileon.es, petkov@cs.rug.nl, enrique.alegre@unileon.es
}

\begin{abstract}
We propose a technique to compute the fraction of boar spermatozoid heads which present an intracellular density distribution pattern hypothesized as normal by veterinary experts. This approach offers a potential for digital image processing estimation of sperm capacitation which can substitute expensive staining techniques. We extract a model distribution from a training set of heads assumed as normal by veterinary experts. We also consider two other training sets, one with heads similar to the normal pattern and another formed by heads that substantially deviate from that pattern. For each spermatozoid head, a deviation from the model distribution is computed. This produces a conditional probability distribution of that deviation for each set. Using a set of test images, we determine the fraction of normal heads in each image and compare it with the result of expert classification. This yields an absolute error below 0.25 in the $89 \%$ of the samples.
\end{abstract}

\section{Introduction}

In the last years, digital image processing and analysis are used for computer assisted evaluation of semen quality with therapeutic goals or to estimate its fertility by means of spermatozoid motility and morphology.

Boar artificial insemination presents more advantages than the natural one: reduction of the number of boars in a farm, maximization of genetic improvements, homogeneous production lots, fertility control of males, and also savings in time and work. Sperm quality analysis is the basic means to avoid infertility problems and to identify boars with the best reproductive features. Generally, four factors are considered to evaluate boar sperm quality: concentration, motility, morphology and acrosome integrity [1]. For instance, if a given sample contains more than $30 \%$ of abnormal spermatozoa, the fertility will be reduced. Computer programs are essential tools in such an evaluation because of the complexity of sperm quality estimation. Digital image analysis can be used to assess this problem.

Fourier descriptors and neural nets yield classification error rates similar to the results obtained by experts in evaluation of human spermatozoid head morphology [2].

A. Gagalowicz and W. Philips (Eds.): CAIP 2005, LNCS 3691, pp. 88-95 2005.

(C) Springer-Verlag Berlin Heidelberg 2005 
Most of the commercial systems in this area are based on motility measures (Hobson Tracking and Mika Medical) [3] or shape abnormalities (Cell-Morf of Motion Analysis) [4]. Hamilton-Thorn combines both features and has a specific module for boar semen [5]. Several morphometric measures are computed by the Sperm Class Analyzer of Microptic [6]. However, some disadvantages are encountered due to the specific design for human spermatozoa. Also aspects like density distribution or intracellular texture are not considered.

Various features are deployed in spermatozoid assessment with digital images, such as cellular motility [7], head abnormalities and distal or proximal droplets. Acrosome integrity and plasma membrane integrity determine the sperm viability because their enzymes take part in the oocyte penetration process. For instance, a pear shaped head, acrosome lifting or a detached acrosome are abnormalities that cause fertility reduction. Spermatozoid heads present a variety of cellular textures that are determined by their corresponding cytoplasmic densities. New research is directed towards finding a correlation between certain patterns of intracellular density distribution and semen fertility. In this approach, veterinary experts first assume that a certain intracellular density distribution is characteristic of healthy cells. Then the fraction of spermatozoid heads in a sample which have intracellular distributions that are sufficiently similar to the assumed model distribution is determined. Applying traditional techniques as vital and fluorescent stains, experts assess the sperm capacitation of such a sample, and try to find a correlation between the above mentioned fraction and semen fertility. The goal is to find a pattern of intracellular density distribution, such that the fraction of sperm heads that exhibit such a pattern has high correlation with the semen fertility as determined by traditional techniques. If successful, this approach can lead to the substitution of expensive staining techniques for fertility evaluation by inexpensive image analysis techniques.

In the current work, we analyse grey-level images of boar semen samples obtained with a phase-contrast microscope, Fig. 1h. More specifically, we study the intracellular density distributions of the spermatozoid heads. Using a training set of images of heads that have been hypothesized by an expert to be "normal", we create a model intracellular density distribution and use it to estimate the fraction of heads in a sample that are sufficiently similar to the model distribution. The goal is to determine automatically the fraction of heads that match an expert's idea of how a normal healthy cell should look like.

In Section 2 we present the methods we have used and the obtained results. Discussion and conclusions are given in Section 3 .

\section{Methods and Results}

\subsection{Image Acquisition, Preprocessing and Head Segmentation}

Fresh boar semen sample images of size $1600 \times 1200$ pixels were captured using a digital camera connected with a phase-contrast microscope at $\times 40$ magnification. They are converted to grey-level images. Each image presents a variable number of spermatozoa whose heads are in different orientations just as tails withouth head, agglutinated heads 
and debris. Using morphological closing, holes in the contours of the heads are filled and the spermatozoid tails are removed. In a next segmentation stage, spermatozoid heads are separated from the background deploying Otsu's method to find a threshold that separates the heads from the background [8]. Heads near the boundary of the image as well as the ones with an area smaller than an experimental obtained value of $45 \%$ of the average head area are not considered. Fig. 1 $\mathrm{b}$ shows a typical image obtained by the above mentioned preprocessing and segmentation.

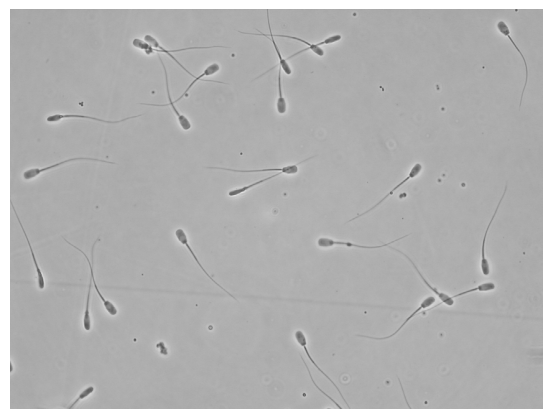

(a)

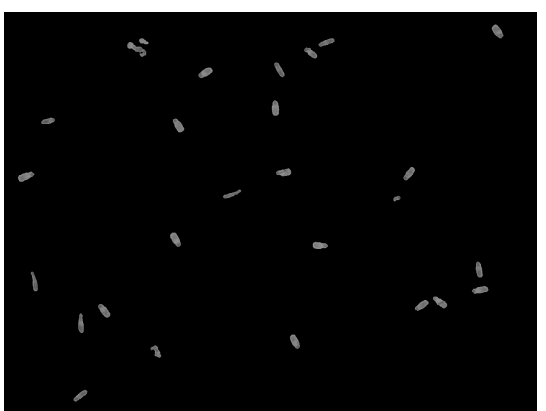

(b)

Fig. 1. (a) Sample image of boar sperm using a phase-contrast microscope. (b) Image obtained after preprocessing and segmentation. The spermatozoid heads are grey-level distributions on a black background.

\subsection{Head Orientation and Re-scaling}

Taking into consideration that a spermatozoid head presents an oval shape, for each of the spermatozoid heads in an image (Fig. 2a), we determine a major and a minor axis of the ellipse that fits into the head by applying principal component analysis. Subsequently, we consider the grey-level distribution in the head in these (head-specific) principal component coordinates. In practice, we rotate the head image so that the major and minor axes of the concerned ellipse coincide with the $x$ and $y$ axes, respectively, Fig. 2 b. According to the empirical measures, a normal boar spermatozoid head takes an oval shape which is from 4 to $5 \mu \mathrm{m}$ wide and from 7 to $10 \mu \mathrm{m}$ long. We re-scale all head images to size $19 \times 35$ pixels. Next, for each head we consider the $2 \mathrm{D}$ function that is defined by the grey levels of those pixels of the head that lie in the fitting ellipse with a minor axis of 19 pixels and a major axis of 35 pixels, Fig. 2 r.

\subsection{Brightness and Contrast Normalization}

Sample images contain heads with diverse intracellular distributions. Three areas can be distinguished in a head image: a darker region which corresponds to the post nucleus cap, an intermediate light area, and the acrosome that covers the nucleus region. However, the contrast between the regions and the average head brightness are not the same 


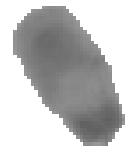

(a)

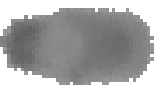

(b)

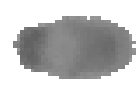

(c)

Fig. 2. (a) Image of a spermatozoid head obtained after preprocessing and segmentation. (b) Rotated head image. (c) 2D grey-level distribution defined in an ellipse fitting in a head image re-scaled to a size of $19 \times 35$.

across different images. To deal with that, we carry out a linear transform on the greylevels of the 2D function of each head, such that after this transform the 2D functions of all heads have the same mean and standard deviation. More precisely, let $f(x, y)$ be the 2D grey-level function defined on a region $S$ enclosed by an ellipse with main axes 19 and 35 pixels. We transform the function $f(x, y)$ into a function $g(x, y)$ defined on $S$ by:

$$
g(x, y)=a f(x, y)+b,
$$

where the coefficients $a$ and $b$ are defined as follows:

$$
a=\frac{\sigma_{g}}{\sigma_{f}}, \quad b=\mu_{g}-a \mu_{f} .
$$

The mean $\mu_{f}$ and the standard deviation $\sigma_{f}$ of $f$ are computed from $f$ and the mean $\mu_{g}$ and the standard deviation $\sigma_{g}$ of $g$ are fixed to $\mu_{g}=100$ and $\sigma_{g}=8$ since the spermatozoid head images assumed as "potentially normal" by experts take around those values for their means and standard deviations.

\subsection{Definition of a Model Head Intensity Distribution}

Next, we compute a model 2D intensity distribution function $m(x, y)$ as an average of a given number of 2D intensity distribution functions obtained from the images of heads that have been hypothesized to be "potentially normal" by experts, Fig. 3a. Such heads are characterized by an appropriate intracellular density distribution according to the regions: dark post nucleus cap, light intermediate area and slightly darker acrosome. Let $g_{i}(x, y), i=1 \ldots n$, be $n$ such functions that were obtained from images of normal heads by applying the above given pre-processing steps of re-scaling and contrast and brightness normalization. In our experiments we took the images of $n=34$ such heads that form our "model" training set $M$. We define the model 2D intensity distribution function $m(x, y)$ as a pixel-wise average of these functions (Fig. 4):

$$
m(x, y)=\frac{1}{n} \sum_{i=1}^{n} g_{i}(x, y)
$$



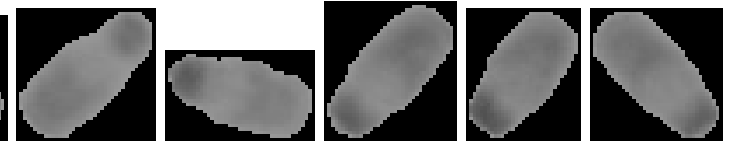

(a)
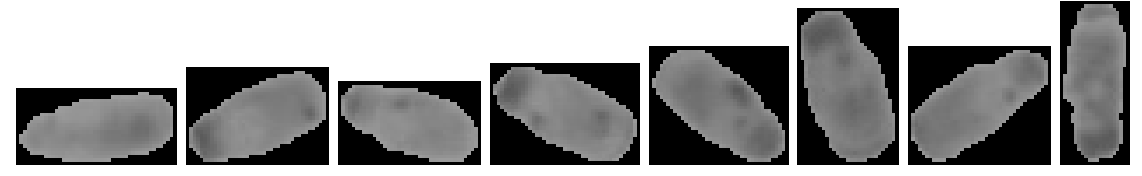

(b)

Fig. 3. Examples of heads that were classified by an expert as having distributions that are (a) similar and (b) not similar to an assumed normal density distribution

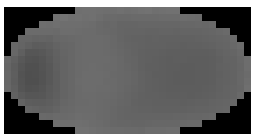

Fig. 4. Intensity map of the model intensity distribution function obtained as an average of the intensity distributions of a number of heads that were assumed to be "potentially normal" by an expert

We also compute the standard deviation $\sigma(x, y)$ in each pixel inside the ellipse to assess the variability of the grey-levels for each point

$$
\sigma(x, y)=\sqrt{\sum_{i=1}^{n} \frac{\left(g_{i}(x, y)-m_{i}(x, y)\right)^{2}}{n}} .
$$

\subsection{Measure of Deviation from the Model Distribution}

We now consider a set of microscopic images of boar semen samples and isolate a number of heads according the above described segmentation method. A re-scaled and normalized intensity distribution function can be computed for each segmented head. Let $g(x, y)$ be the function that represents one of the heads observed in the image. We now compute a measure of deviation $d$ of this function from the model function $m(x, y)$ using the $L_{\infty}$ norm:

$$
d=\max \left(\frac{|g(x, y)-m(x, y)|}{\sigma(x, y)}\right) .
$$

We compute the value of this measure of deviation from the model intensity distribution for every head in an image. Different values are computed for different heads. Fig. $5 \mathrm{a}$ presents a histogram of the values that were computed for a group of 44 heads that were hypothesized to be normal by an expert from the isolated head images (Fig. 3 $\mathrm{k}$ ). These 
heads form our "normal" training set $N$ and yield values for $d$ from 2 to 5.2. This histogram defines a conditional probability distribution $P(d \mid n)$ of observing deviation value $d$ for a normal head. Similarly, Fig. $5 \mathrm{~b}$ presents a histogram of the values that were computed for a group of 82 heads (from a "not-normal" or "bad" training set B) that were classified as "not-normal" by an expert (Fig. 3b). The obtained values of $d$ for each head image of such set $B$ fall in the range $[3,15]$. This histogram defines a conditional probability distribution $P(d \mid b)$ of observing deviation value $d$ for a not-normal head.

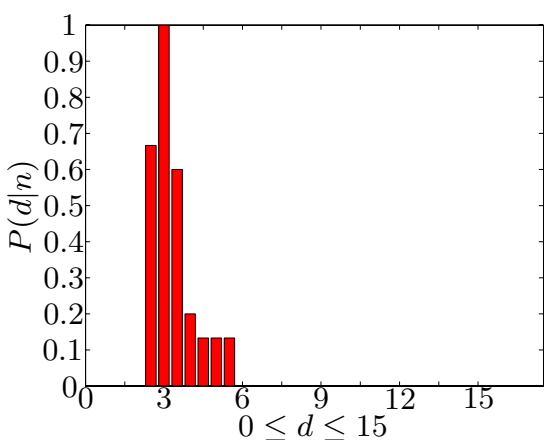

(a)

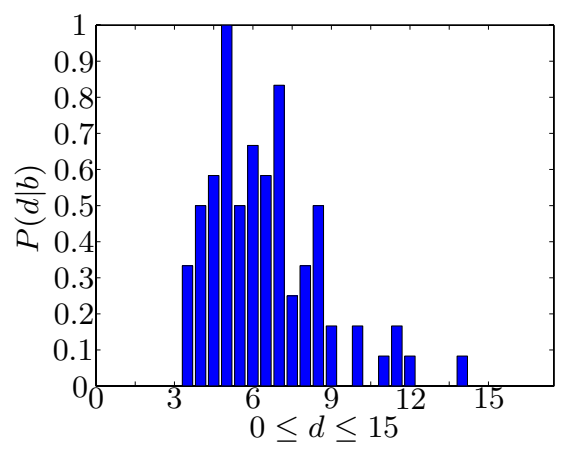

(b)

Fig. 5. Histograms of the values of the deviation from the model function for (a) normal and (b) not-normal heads. These histograms can be seen as conditional probability distributions $P(d \mid n)$ and $P(d \mid b)$, respectively.

\subsection{Estimation of the Fraction of Normal Heads in an Image}

Considering a new boar semen sample, we next estimate the fraction of normal heads. For this purpose, a deviation value $d$ is computed for each head in a microscopic image of the semen sample and a histogram of the observed values is built. This histogram defines a distribution $P(d)$ for which in theory holds:

$$
P(d)=P(d \mid n) P_{n}+P(d \mid b)\left(1-P_{n}\right) .
$$

where $P(d \mid n)$ and $P(d \mid b)$ are the above defined conditional probabilities of observing deviation value $d$ for a normal and a not-normal head, respectively, and $P_{n}$ and $1-P_{n}$ are the probabilities that a given head is normal or not-normal, respectively. In the above equation, $P(d), P(d \mid n)$ and $P(d \mid b)$ are considered as known and $P_{n}$ as unknown. This equation defines an overdetermined system of linear equations for $P_{n}$ that contains one equation for each histogram bin of $d$. The approximate solution to this system according to the least squares method is given by:

$$
P_{n}=\frac{\sum_{d}(P(d)-P(d \mid b))(P(d \mid n)-P(d \mid b))}{\sum_{d}(P(d \mid n)-P(d \mid b))^{2}} .
$$

Using the distribution $P(d)$ for the considered sample and the conditional probabilities $P(d \mid n)$ and $P(d \mid b)$ pre-computed on the training sets $N$ and $B$ of sample images, 
the above formula is an effective means to compute the fraction $P_{n}$ of normal heads in a sample. Note that we estimate this fraction without having to classify each head separately as normal or not normal.

\subsection{Experimental Results}

A test set $T$ of 100 images of different samples of boar sperm was considered. After preprocessing, segmentation, brightness and contrast normalization, each head was described by a 2D grey level distribution function and a deviation of this function from the model distribution function as defined above was computed. The heads obtained from a given sample yield a histogram $P(d)$ of the deviation from the model for that sample. Then, the fraction $P_{n}$ of heads with a normal intracellular distribution was evaluated for each test image according to the method described above. The obtained 100 values are illustrated by a box-and-whisker diagram shown in Fig. 6a. These values were compared with the values of the fraction $P_{e}$ of normal heads in the concerned images as determined by an expert (Fig. 60). The values of the absolute error $P_{n}-P_{e}$ computed for the different test images are illustrated by the box-and-whisker diagram shown in Fig. 66. The absolute error is below 0.25 for 89 of the 100 test images, between 0.25 and 0.32 for another 8 test images and below 0.47 for the 3 remaining images.

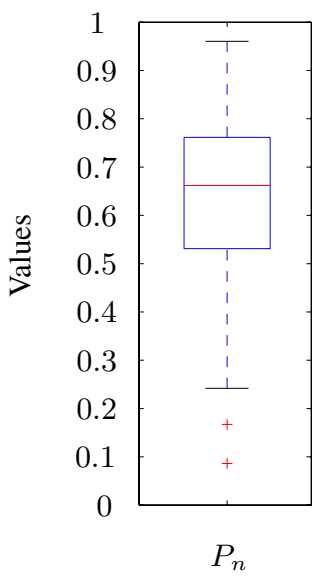

(a)

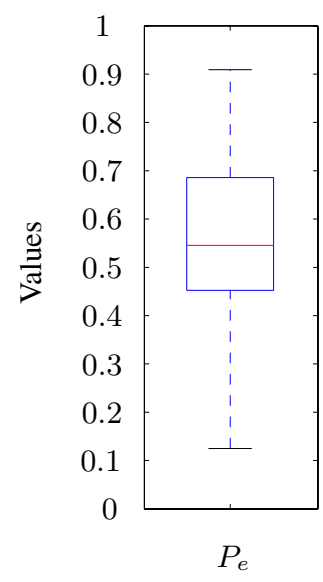

(b)

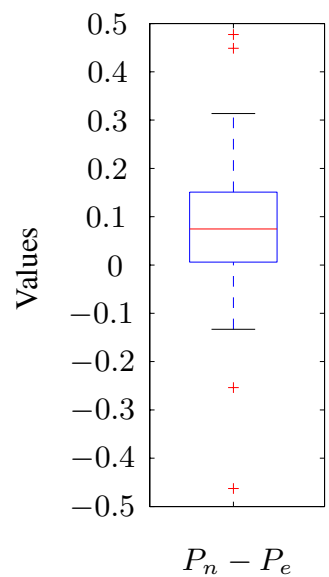

(c)

Fig. 6. (a) Box-and whisker diagrams of the values of the fraction of normal heads determined by means of the proposed method, and (b) by veterinary experts and (c) absolute error of the method in comparison with the experts

\section{Discussion and Conclusions}

We proposed a novel approach to the analysis of images of boar spermatozoid heads in order to describe and classify them by means of their intracellular distribution. Using a training set $M$ of spermatozoid head images that were hypothesized by a veterinary 
expert as potentially "normal", we compute a model intracellular distribution for such a head. For each head in an image we compute a value of dissimilarity of its intracellular distribution to the model distribution. The histogram of dissimilarity values obtained for the intracellular distributions of normal heads is different from the histogram obtained for the distributions of heads that are considered by an expert as potentially not normal. To build these histograms we use two other training sets $(N$ and $B)$. We use the two histograms to estimate the fraction of heads in a semen sample image that would be classified as "potentially normal" by an expert. The absolute error of our method compared to a human expert is less than 0.25 in $89 \%$ of the sample images. That is an encouraging result because this work is the first that uses cellular density information instead of morphological features. Note that our algorithm gives systematically an overestimation of the concerned fraction. Our future work will be directed towards reducing this error. We will also explore the potential of single head classification in which first each head is classified individually as normal or not normal and the results for all heads in a sample image are used to determine the concerned fraction of normal cells.

The absolute error defined above is not the final criterion for the performance of our method because the classification by a human expert can differ from one session to another and across experts. It is more important that, once the algorithm is trained to evaluate the fraction of potentially normal heads in a sample, this fraction is correlated with the sperm vitality of that sample as determined by means of staining techniques. The potential of our approach in that respect will next be tested in veterinary praxis.

\section{References}

1. Thurston, L., Holt, W., Watson, P.: Post-thaw functional status of boar spermatozoa cryopreserved using three controlled rate freezers: a comparison. Theriogenology 60 (2003) 101-113

2. Linneberg, C., Salamon, P., Svarer, C., Hansen, L.: Towards semen quality assessment using neural networks. In: Proc. IEEE Neural Networks for Signal Processing IV. (1994) 509-517

3. Verstegen, J., Iguer-Ouada, M., Onclin, K.: Computer assisted semen analyzers in andrology research and veterinary practice. Theriogenology 57 (2002) 149-179

4. Gravance, C., Garner, D., Pitt, C., Vishwanath, R., Sax-Gravance, S., Casey, P.: Replicate and technician variation associated with computer aided bull sperm head morphometry analysis (asma). International Journal of Andrology 22 (1999) 77-82

5. PIC: Artificial insemination: Semen processing and quality control. Artificial Insemination 1 (2003)

6. Quintero, A., Rigaub, T., Rodríguez, J.: Regression analyses and motile sperm subpopulation structure study as improving tools in boar semen quality analysis. Theriogenology 61 (2004) $673-690$

7. Robles, V., Alegre, E., Sebastián, J.: Tracking algorithms evaluation in feature points image sequences. In Campilho, A., Kamel, M., eds.: Image Analysis and Recognition: International Conference ICIAR 2004, Porto, Portugal, September 29 - October 1, 2004, Proceedings, Part II. Volume 3212 of Lecture Notes in Computer Science. Springer-Verlag Heidelberg (2004) 589-596

8. Alegre, E., Sánchez, L., Aláiz, R., Dominguez, J.: Utilización de momentos estadísticos y redes neuronales en la clasificación de cabezas de espermatozoides de verraco. In: XXV Jornadas de Automática. (2004) 